\title{
Meningitis por criptococo Cryptococcal meningitis
}

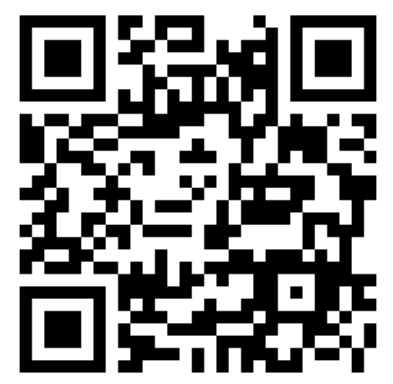

Recibido 26/05/2021
Corregido $16 / 06 / 2021$

${ }^{1}$ Dra. Estefanía Chaves Loaiza Hospital San Carlos, Alajuela, Costa Rica (Di) https://orcid.org/0000-0002-2994-5740

${ }^{2}$ Dr. Johynny Bernarda Solís Solís Hospital San Carlos, Alajuela, Costa Rica (D) https://orcid.org/0000-0002-6253-0707

\section{RESUMEN}

La meningitis criptocócica se trata de una grave infección del sistema nervioso central causada principalmente por las especias de Cryptococcus neoformans y Cryptococcus gatti que ocurre principalmente en pacientes inmunosuprimidos, en especial en pacientes portadores de $\mathrm{VIH}$. Causante de muchas de las muertes relacionadas al SIDA a nivel mundial. El diagnóstico se realiza mediante el aislamiento del hongo en cultivo en líquido cefalorraquídeo o identificación del antígeno criptocócica en análisis de sangre. Es necesario una detección e intervención terapéutica temprana, para mejorar de forma significativa la supervivencia y calidad de vida de los pacientes.

PALABRAS CLAVE: meningitis criptocócica; VIH; inmunosupresión; terapia antirretroviral altamente activa.

\section{ABSTRACT}

Cryptococcal meningitis is a serious infection of the central nervous system caused mainly by Cryptococcus neoformans and Cryptococcus gatti that occurs mainly in immunosuppressed patients, especially in patients with HIV. It causes many AIDS-related deaths worldwide. Diagnosis is made by isolation of the fungus in cerebrospinal fluid culture or identification of cryptococcal antigen in blood tests. Early detection and therapeutic intervention is necessary to significantly improve patient survival and quality of life.

KEYWORDS: meningitis, cryptococcal; HIV; immunosuppression; antiretroviral therapy, highly active.

${ }^{1}$ Médica general, graduada de la Universidad Latina de Costa Rica (U. Latina). Cód. MED15519. Correo: estefania.chaves@yahoo.com 
${ }^{2}$ Médico general, graduado de la Universidad Latina de Costa Rica (U. Latina). Cód. MED13428. Correo: johynny@gmail.com

\section{INTRODUCCIÓN}

La meningitis criptocócica es una de las principales causas de meningitis en muchos países de ingresos bajos y medios, con una alta prevalencia de pacientes infectados por el virus de inmunodeficiencia humana (VIH) con un número cada vez mayor de muertes asociados a esta infección, que también se produce en pacientes sin VIH con algún otro tipo de inmunosupresión.

Corresponde a una infección grave del sistema nervioso central que genera además gran problema de salud pública debido a su alta mortalidad y de discapacidad a largo plazo, por lo que es importante realizar un diagnóstico rápido de la infección junto con intervenciones terapéuticas tempranas, para mejorar la supervivencia y la calidad de vida de estos pacientes.

El objetivo de la presente revisión bibliográfica es lograr reconocer la infección de forma temprana, los métodos diagnósticos disponibles y las opciones farmacológicas actualizadas con mayor evidencia científica para el tratamiento óptimo de la infección.

\section{MÉTODO}

Para el presente artículo de revisión bibliográfica, se revisaron 15 diferentes fuentes bibliográficas que comprenden entre los años 2016 al 2020, seleccionados de bases de datos como Elsevier, Scielo, Oxford Academic, Dialnet, ScienceDirect. Se utilizaron las revistas médicas de Sociedad Neurológica Argentina, revista ecuatoriana de neurología, acta médica peruana, New England Journal of Medicine. Se aplicaron palabras clave como "meningitis criptocócica", "VIH", "inmunosupresión", "terapia antirretroviral altamente activa" y sus homónimos en inglés. Se incluyeron estudios de países como Australia, África, Honduras, Ecuador y Estados Unidos. La mayoría de los artículos de revisión bibliográfica, sin embargo, también se incluyeron estudios poblacionales, donde la muestra más pequeña fue de 299 pacientes y la más grande de 451 pacientes. De la totalidad de los artículos en revisión, 11 de ellos fueron en su idioma original en inglés y 4 de ellos en español.

\section{EPIDEMIOLOGÍA}

La meningitis criptocócica (MC) fue descrita por primera vez en 1890 y en 1905 se reportó el primer caso por este patógeno (1). Con la introducción de la terapia antirretroviral (TAR) para el tratamiento de pacientes con $\mathrm{VIH}$, la incidencia de $\mathrm{MC}$ disminuyó en países de ingresos altos, pero todavía es una de las principales causas de meningitis en muchos países de ingresos bajos y medios donde hay una alta prevalencia de infección por VIH, representando el $15-20 \%$ de toda la mortalidad y acorde a los datos de la organización mundial de la salud (OMS) en el año 2005 alcanzó los 2.3 millones de fallecidos. Además, un número cada vez mayor de muertes asociadas se producen en pacientes sin $\mathrm{VIH}$ en países de ingresos altos $(2,3)$, debido a otras causas como uso prolongado de corticoesteroides, enfermedades autoinmunes, neoplasias, trasplantes, diabetes, cirrosis hepática, insuficiencia renal crónica terminal $(1,4)$.

La tasa de infección en la población inmunocompetente es de aproximadamente $1 / 100.000$, mientras que en pacientes inmunodeprimidos puede alcanzar 5-10\% y puede llegar a $30 \%$ en pacientes con SIDA (4).

En pacientes con VIH causa más de 600.000 muertes al año en todo el mundo (5). Tiene 
una alta tasa de letalidad, que en África subsahariana se estima en un $70 \%$ a los 3 meses (6).

Un $60 \%$ de los pacientes que son $\mathrm{VIH}$ positivos debutan con un episodio clínico de $\mathrm{MC}$, que corresponde a la cuarta causa más común de infecciones graves en pacientes con el virus (7).

La incidencia total de criptococosis en pacientes trasplantados es del $2.8 \%$ y el 53 $72 \%$ de los casos comprometen el sistema nervioso central (SNC) (8).

\section{PATOGENIA}

Es una micosis causada por dos hongos levaduriformes escapsulados del género Cryptococcus, C. neoformans y C. gatti que se adquiere por la inhalación de las levaduras desecadas existentes en la naturaleza.

C. neoformans causa enfermedades en pacientes inmunosuprimidos, mientras de $C$. gatti afecta preferentemente a los inmunocompetentes (7).

Cuando el criptococo ingresa al pulmón no es eliminado de los alveolos ni queda latente por la respuesta inmune mediada por células, sino que se propaga por vía hematógena y entra en el líquido cefalorraquídeo (LCR) por la migración transcelular a través del endotelio microvascular de la barrera hematoencefálica o puede cruzar dentro de las células mononucleares, dicho mecanismo es conocido como el "caballo de Troya" (4).

La patogenicidad se determina por la cápsula que impide la fagocitosis y la actuación del complemento y por la enzima fenil-oxidasa que contribuye al especial neurotropismo del hongo, siendo el (SNC) la localización más frecuente de la infección.

La criptococosis es poco frecuente y afecta preferentemente a criadores de palomas, aficionados de las aves o personal de laboratorio. El habitad del hongo se ha encontrado en el $70 \%$ del excremento de las palomas y en el humano se considera una infección oportunista (4).

\section{MANIFESTACIONES CLÍNICAS}

Las manifestaciones típicas del cuadro meníngeo se dan en menos del $30 \%$ y generalmente se presentan como meningitis subaguda con cefalea que es progresiva en intensidad, fiebre y vómitos, seguido de afectación del nivel de conciencia, crisis epilépticas, desorientación y déficits neurológicos focales $(8,9)$.

La duración de las manifestaciones clínicas tales como la cefalea subaguda y confusión pueden variar desde unos pocos meses hasta incluso veinte años (7).

Esta infección es fatal si no es diagnosticada y tratada a tiempo y aunque fuese revertida exitosamente podría dejar graves secuelas neurológicas, entre las que se destacan ceguera e hidrocefalia (7).

Muchas de las complicaciones son oftálmicas entre las que incluyen: diplopía binocular, parálisis del nervio abducens ya sea monocular o binocular, papiledema, hemorragias peripapilares, atrofia óptima, coroiditis multifocal, entre otros (7).

\section{DIAGNÓSTICO}

El estudio de LCR es el examen esencial para la confirmación de la infección del SNC, mediante el aislamiento del hongo en cultivo, la citoquímica generalmente presenta pleocitosis linfocitaria, hiperproteinorraquia leve e hipoglucorraquia por consumo de glucosa $(4,9)$.

La tinción con tinta china se consideró como una prueba de tamizaje relativamente buena por su buen valor predictivo negativo, es barata y solo se necesita que el personal de laboratorio sepa reconocer las levaduras encapsuladas en el microscopio. Sin embargo, la sensibilidad del $80 \%$ podría 
dejar escapar algunas muestras positivas (7).

A pesar de que el cultivo es el estándar de oro para el diagnóstico, su realización es costosa y requiere de equipamiento e infraestructura, además de la cantidad de tiempo de espera para el resultado (alrededor de 48 horas), en comparación con métodos de tinta china, aglutinación de látex (LA) y ensayo flujo lateral (LFA) es demasiado largo (7).

Se puede hacer un diagnóstico presuntivo mediante una prueba de antígeno criptocócico (CrAg) positivo en suero o LCR, la positividad de la prueba tiene una alta sensibilidad y especificidad que es superior al $99 \%(4)$.

Por otro lado, la LA es más rápida, fácil, específica y más importante sensible en comparación con otros métodos convencionales, pero se recomienda más bien el uso de la prueba de inmunocromatografía de LFA CrAg como tamizaje en $\mathrm{VIH}+$ con recuento de CD4 $\leq$ $100 / \mu \mathrm{L}$, pues el costo-beneficio es justo (7).

\section{TAMIZAJE DE ANTÍGENO CRIPTOCÓCICO}

El criptococo contiene un polisacárido capsular, conocido como $\mathrm{CrAg}$, que puede detectarse en la sangre semanas o meses antes de la aparición de la MC por medio de una muestra en suero o plasma mediante procedimientos de LA o LFA (10).

Las personas con antigenemia criptocócica corren un alto riesgo de desarrollar MC si no reciben tratamiento.

No está claro la progresión y el momento en que se pasa de una infección asintomática a la MC. Se estima que la prevalencia de antigenemia criptocócica es un $6 \%$ en pacientes con $\mathrm{VIH}+\mathrm{y}$ recuentos de células CD4 < 100 células $/ \mu \mathrm{L}$ y la positividad del CrAg aumenta significativamente con las cargas virales más altas por lo que la OMS recomienda examinar a todas las personas infectadas por el VIH sin TAR con estos valores de recuentos celulares (10-13).

En pacientes con CrAg positivos se les deberá ofrecer realizar una punción lumbar para evitar que los pacientes clínicamente asintomáticos con evidencia de meningitis en LCR reciban un tratamiento antifúngico subóptimo $(10,14)$.

\section{HALLAZGOS RADIOLÓGICOS}

Las imágenes de la infección criptocócica en el SNC pueden ser variadas, que se relacionan principalmente con la propensión del organismo a la propagación hematógena y la diseminación a lo largo de los espacios perivasculares en la base del cerebro (4).

Cuando hay afectación meníngea por lo general es más prominente en la base del cerebro por lo que en este caso la TC sigue siendo menos sensible que la RM, sobre todo para identificar realce meníngeo basal que se observaría en estos casos (4).

Algunos de los hallazgos radiológicos dependerán del sitio de afectación de la infección, por ejemplo, la visualización de criptococomas cuando hay afección de plexos coroideos o parenquimatosa, pseudoquistes en el compromiso del espacio perivascular ya que agranda los espacios perivasculares, además se pueden observar calcificaciones puntiformes en leptomeninges y parénquima (4).

Es importante destacar que la tomografía computarizada (TC) y la resonancia magnética (RM) convencional pueden ser normales hasta en un $50 \%$ de los casos o simplemente mostrar una atrofia cortical inespecífica en este grupo de pacientes y no existe una imagen cerebral patognomónica de criptococosis $(4,15)$.

\section{TRATAMIENTO}

El tratamiento de primera línea según las guías para estos pacientes corresponde a dos fases, una de inducción con anfotericina 
B a una dosis de 0.7-1.0 $\mathrm{mg} / \mathrm{kg} /$ día más flucitosina $100 \mathrm{mg} / \mathrm{kg} /$ día por 2 semanas y luego una fase de consolidación con fluconazol $400-800 \mathrm{mg} /$ día por 8 semanas y terapia de mantenimiento con fluconazol 200 mg al día por 6-12 meses (4,13-15)

La anfotericina $B$ requiere de una administración intravenosa y un estrecho control de laboratorio y se asocia a flebitis, infecciones secundarias, anemia y deterioro renal (6).

La flucitosina con frecuencia no se encuentra disponible, principalmente en lugares de escasos recursos debido a su alto costo y efectos secundarios que limitan su uso (16). Este fármaco se asocia a una eliminación más rápida de la infección, por lo que se necesita la disponibilidad generalizada de flucitosina como parte esencial de los programas mundiales para reducir la mortalidad.

El fluconazol si es fácilmente disponible, se asocia con bajas tasas de efectos adversos y tiene buena penetración dentro del LCR, pero en monoterapia para el tratamiento de la $\mathrm{MC}$ se asocia a pobres resultados. Debido a su perfil de ser seguro, bajo costo y disponibilidad hace que sea una atractiva alternativa a la flucitosina en conjunto con anfotericina $\mathrm{B}$ y es recomendada en las guías de tratamiento. Sin embargo, se debe indicar la dosis de anfotericina $B$ de 1 $\mathrm{mg} / \mathrm{kg} /$ día y fluconazol 800-1200 mg por día por 2 semanas y luego el mantenimiento con fluconazol como descrito previamente, para un mejor resultado en la eliminación de la levadura. $(5,16)$

Además, en pacientes con detección de CrAg se ha recomendado tratamiento preventivo con fluconazol a dosis de $800 \mathrm{mg}$ diarios por 2 semanas, seguidos de $400 \mathrm{mg}$ diarios durante 2 meses y luego $200 \mathrm{mg}$ diarios en espera de reconstitución inmunológica (14). En pacientes con antigenemia positiva y con evidencia de meningitis en LCR deben ser tratados con la terapia combinada antifúngica de anfotericina B más flucitosina o fluconazol y el fluconazol en monoterapia debe restringirse a aquellos sin evidencia de MC en el LCR (10).

La terapia TAR se retrasa 2 semanas en pacientes asintomáticos con CrAg positivo y de 4 a 6 semanas si se diagnostica MC (14). Con respecto al uso de glucocorticoides adyuvantes que reducen la mortalidad en pacientes otras formas de meningitis, no reduce la mortalidad en pacientes con MC asociada al VIH y se asoció con más acontecimientos adversos y discapacidad por lo que se recomienda su uso $(5,13)$.

\section{CONCLUSIONES}

A pesar de que con la introducción de la TAR, la incidencia de MC disminuyó en países de ingresos altos, todavía hay una gran cantidad de pacientes en países de bajos recursos que acuden a la consulta con recuentos celulares de CD4 bajos y corresponde a un importante factor de riesgo para desarrollar MC, por lo que es importante el tamizaje de éstos pacientes para detectar antigenemia criptocócica y brindar un tratamiento preventivo o ya sea detectar los casos de MC asintomática con el fin de evitar dar tratamientos subóptimos y evitar aumentar la mortalidad o discapacidad a largo plazo.

Es necesario la disponibilidad generalizada de la flucitosina como parte esencial de los programas mundiales para reducir la mortalidad relacionada con el $\mathrm{VIH}$, así como la investigación continua de nuevas terapias farmacológicas.

\section{Los autores declaran no tener conflicto de interés.}

\section{REFERENCIAS}

1. Barquero M. Meningitis por cryptococcus neoformans: Revista médica de Costa Rica y Centroamérica. 2016, LXXIII (619) 205 - 208. [accesado 17 Mayo 2021]. Disponible 
https://www.medigraphic.com/pdfs/revmedcosce n/rmc-2016/rmc162c.pdf

2. Pasquier E, Kunda J, De Beaudrap P, Loyse A, Temfack E, Molloy S et al. Long term mortality and disability in Cryptococcal Meningitis: a systematic literature review. Clinical Infectious Diseases [Internet]. 2017 [cited 4 April 2020];. Available from:

https://academic.oup.com/cid/article/66/7/1122/4 344944? searchresult=1

3. Pullen $M$, Hullsiek $K$, Rhein J, Musubire $A$, Tugume L, Nuwagira E et al. Cerebrospinal Fluid Early Fungicidal Activity as a Surrogate Endpoint for Cryptococcal Meningitis Survival in Clinical Trials. Clinical Infectious Diseases [Internet]. 2020 [cited 4 April 2020];. Available from: https://academic.oup.com/cid/advance-articleabstract/doi/10.1093/cid/ciaa016/5698219?redire ctedFrom=fulltext

4. Offiah C, Naseer A. Spectrum of imaging appearances of intracranial cryptococcal infection in HIVIAIDS patients in the anti-retroviral therapy era. Clinical Radiology [Internet]. 2016 [cited 9 August 2020];71(1):9-17. Available from: https://www.sciencedirect.com/science/article/abs /pii/S000992601500392X

5. Beardsley J, Wolbers M, Kibengo F, Ggayi A, Kamali A, Cuc $\mathrm{N}$ et al. Adjunctive Dexamethasone in HIV-Associated Cryptococcal Meningitis. New England Journal of Medicine [Internet]. 2016 [cited 5 April 2020];374(6):542-554. Available from: https://www.nejm.org/doi/full/10.1056/NEJMoa15 $\underline{09024}$

6. Molloy S, Kanyama C, Heyderman R, Loyse A, Kouanfack C, Chanda D et al. Antifungal Combinations for Treatment of Cryptococcal Meningitis in Africa. New England Journal of Medicine [Internet]. 2018 [cited 5 April 2020];378(11):1004-1017. Available from: https://www.nejm.org/doi/full/10.1056/NEJMoa17 10922

7. Barcia Castilla V, Sanchez Giler S. Utilidad de la prueba de tinta china como tamizaje para meningitis por Cryptococcus spp / Utility of indian ink as screening for meningitis by Cryptococcus spp. Ciencia Unemi [Internet]. 2016 [cited 3 April 2020];9(20):63. Available from: https://dialnet.unirioja.es/servlet/articulo?codigo= 5774761

8. Pontello N, Gleichgerrcht E, Facundo M, Sinay V. Criptococosis meníngea en inmunosuprimidos: rol del síndrome inflamatorio de reconstitución inmune. Neurología Argentina [Internet]. 2012 [cited 19 February 2020];4(1):31-34. Available from: $\quad$ https://www.elsevier.es/es-revistaneurologia-argentina-301-articulo-criptococosismeningea-inmunosuprimidos-rol-del$\underline{\mathrm{S} 1853002811000668}$
9. Rivero D, Scherle C, Loza G, Pillajo G, Pernas Y. Meningitis Criptocócica. Diferentes Contextos Clínicos y Complicaciones. Serie de 7 Casos. Revista Ecuatoriana de Neurología. 2019 (citado el 19 de febrero de 2020). Vol. 28, №2. Disponible en: $\quad$ http://revecuatneurol.com/wpcontent/uploads/2019/10/2631-2581-rneuro-2802-00028.pdf

10. Temfack E, Bigna J, Luma H, Spijker R, Meintjes $\mathrm{G}$, Jarvis $\mathrm{J}$ et al. Impact of Routine Cryptococcal Antigen Screening and Targeted Preemptive Fluconazole Therapy in Antiretroviral-naive Human Immunodeficiency Virus-infected Adults With CD4 Cell Counts <100/ $\mu \mathrm{L}$ : A Systematic Review and Meta-analysis. Clinical Infectious Diseases [Internet]. 2018 [cited 4 April 2020];68(4):688-698. Available from: https://academic.oup.com/cid/article/68/4/688/50 55339?searchresult=1

11. Mpoza E, Rajasingham R, Tugume L, Rhein J, Nabaggala M, Ssewanyana I et al. Cryptococcal Antigenemia in HIV therapy-experienced Ugandans with Virologic Failure. Clinical Infectious Diseases [Internet]. 2019 [cited 4 April 2020];. Available from: https://academic.oup.com/cid/advancearticle/doi/10.1093/cid/ciz1069/5611261?searchr esult=1

12. Ssebambulidde $K$, Bangdiwala A, Kwizera $R$, Kandole T, Tugume L, Kiggundu $\mathrm{R}$ et al. Symptomatic Cryptococcal Antigenemia Presenting as Early Cryptococcal Meningitis With Negative Cerebral Spinal Fluid Analysis. Clinical Infectious Diseases [Internet]. 2018 [cited 4 April 2020];68(12):2094-2098. Available from: https://academic.oup.com/cid/articleabstract/68/12/2094/5106758?redirectedFrom=ful Itext

13. World Health Organization. The diagnosis, prevention and management of cryptococcal disease in hiv-infected adults, adolescents and children. Netherlands: World Health Organization ; 2018.

14. Wake R, Govender N, Omar $T$, Nel C, Mazanderani A, Karat A et al. Cryptococcalrelated Mortality Despite Fluconazole Preemptive Treatment in a Cryptococcal Antigen Screen-andTreat Program. Clinical Infectious Diseases [Internet]. 2019 [cited 4 April 2020];. Available from: https://academic.oup.com/cid/advancearticle-

abstract/doi/10.1093/cid/ciz485/5513150?redirect edFrom=fulltext

15. Trejo-Espino A, Ramírez-Izcoa A, AlvaradoRivera S, Godoy-Mejía C, Valenzuela-Castillo R. Meningoencefalitis por Cryptococcus neoformans en adolescente con desnutrición. ACTA MEDICA PERUANA [Internet]. 2016 [cited 19 February 
2020];33(3):232. Available from: http://www.scielo.org.pe/pdf/amp/v33n3/a11v33n 3.pdf

16. Day J, Chau T, Wolbers M, Mai P, Dung N, Mai N et al. Combination Antifungal Therapy for Cryptococcal Meningitis. New England Journal of Medicine [Internet]. 2013 [cited 19 February 2020];368(14):1291-1302. Available from: https://www.nejm.org/doi/full/10.1056/NEJMoa11 $\underline{10404}$ 